\title{
Dose Escalation Improves Outcome in Stereotactic Body Radiotherapy for Pulmonary Oligometastases from Colorectal Cancer
}

\author{
KEIICHI JINGU ${ }^{1}$, YUKINORI MATSUO ${ }^{2}$, HIROSHI ONISHI ${ }^{3}$, TAKAYA YAMAMOTO ${ }^{1}$, MASAHIKO AOKI ${ }^{4}$, \\ YUJI MURAKAMI ${ }^{5}$, HIDEOMI YAMASHITA ${ }^{6}$, HISAO KAKUHARA $^{7}$, KENJI NEMOTO $^{8}$, TORU SAKAYAUCHI $^{9}$, \\ MASAHIKO OKAMOTO ${ }^{10}$, YUZURU NIIBE ${ }^{11}$, YASUSHI NAGATA ${ }^{5}$ and KAZUHIKO OGAWA ${ }^{12}$ \\ ${ }^{1}$ Department of Radiation Oncology, Graduate School of Medicine, Tohoku University Sendai, Japan; \\ ${ }^{2}$ Department of Radiation Oncology and Image-applied Therapy, \\ Graduate School of Medicine, Kyoto University, Kyoto, Japan; \\ ${ }^{3}$ Department of Radiology, Graduate School of Medicine, Yamanashi University, Yamanashi, Japan; \\ ${ }^{4}$ Department of Radiology and Radiation Oncology, \\ Graduate School of Medicine, Hirosaki University, Hirosaki, Japan; \\ ${ }^{5}$ Department of Radiation Oncology, Graduate School of Medicine, Hiroshima University, Hiroshima, Japan; \\ ${ }^{6}$ Department of Radiology, Graduate School of Medicine, The University of Tokyo, Tokyo, Japan; \\ ${ }^{7}$ Department of Radiology, School of Medicine, Iwate Medical University, Morioka, Japan; \\ ${ }^{8}$ Department of Radiology, Graduate School of Medicine, Yamagata University, Yamagata, Japan; \\ ${ }^{9}$ Department of Radiation Oncology, Osaki Citizen Hospital, Furukawa, Japan; \\ ${ }^{10}$ Department of Radiation Oncology, Graduate School of Medicine, Gunma University, Maebashi, Japan; \\ ${ }^{11}$ Department of Radiology, Toho University Omori Medical Center, Tokyo, Japan; \\ ${ }^{12}$ Department of Radiation Oncology, Graduate School of Medicine, Osaka University, Osaka, Japan
}

\begin{abstract}
Aim: To evaluate the efficacy of stereotactic body radiotherapy (SBRT) for pulmonary metastasis from colorectal cancer. Patients and Methods: Data for 104 lesions from 93 patients who underwent SBRT for pulmonary oligometastases from colorectal cancer at ten Institutions were retrospectively analyzed. Toxicity was graded according to the Common Terminology Criteria for Adverse Events v4.0. Results: The median calculated biological effective dose using the linear-quadratic model with $\alpha / \beta$ of $10 \mathrm{~Gy}$ (BED10) was 105.6 Gy. Adjuvant chemotherapy after SBRT was performed in 47 patients. The median observation period was 28 months. The 3-and 5-year local control rates were $65.2 \%$ and $56.2 \%$, respectively. The 3-and 5-year overall survival rates were $55.9 \%$ and $42.7 \%$, respectively. Only two patients had grade 3 radiation pneumonitis. In multivariate analysis for local control, primary site, age,
\end{abstract}

Correspondence to: Keiichi Jingu, MD, Ph.D., Department of Radiation Oncology, Tohoku University Graduate School of Medicine, 1-1 Seiryochou, Aoba-ku, Sendai 980-8574, Japan. Tel: +81 227177312, Fax: +81 227177316, e-mail: kjingu-jr@rad.med.tohoku.ac.jp

Key Words: Oligometastases, colorectal cancer, SBRT, dose-escalation. adjuvant chemotherapy after SBRT and BED10 were selected as prognostic factors. Conclusion: Dose escalation and adjuvant chemotherapy might improve local control in SBRT for pulmonary oligometastases from colorectal cancer.

Lung metastases occur in 7-10\% of patients with colorectal cancer $(1,2)$. The term oligometastases, which means fewer than five metastatic lesions, has become popular. Pulmonary metastases from colorectal cancer should be resected as much as possible (3); however, stereotactic body radiotherapy (SBRT) for pulmonary oligometastases is known to be almost as effective as metastasectomy. However, some institutions, including our hospitals, have shown that the local control rate for pulmonary oligometastases from colorectal cancer by SBRT was worse than that for oligometastases from other cancer types by $\operatorname{SBRT}(4,5)$.

The efficacy and safety of SBRT for primary lung cancer have been established. SBRT is an alternative treatment method for early lung cancer. In the present study, we retrospectively reviewed data for patients with pulmonary metastasis from colorectal cancer who were treated by SBRT in Japanese hospitals in order to determine the efficacy and to clarify the prognostic factors for local control and overall survival. 
Table I. Patient characteristics.

\begin{tabular}{lc}
\hline Characteristic & Value \\
\hline Gender, $\mathrm{n}$ & \\
Male & 60 \\
Female & 33 \\
Age, years & \\
$\quad$ Median & 69 \\
Range & $34-79$ \\
Primary clinical stage, $\mathrm{n}$ & \\
I-II & 26 \\
III-IV & 54 \\
Unknown & 13 \\
Primary site, $\mathrm{n}$ & \\
Colon & 48 \\
Rectum & 45 \\
Disease-free interval, months & \\
Median & 18.2 \\
Range & $0-112.5$ \\
Performance status, $\mathrm{n}^{\S}$ & \\
0-1 & 89 \\
2 & 5 \\
Tumor diameter, mm & \\
Median & 15 \\
Range & $4-37$ \\
CEA, ng/ml & \\
Median & 4.5 \\
Range & $0-175.6$ \\
\hline
\end{tabular}

CEA: Carcinoembryonic antigen. §According to the Eastern Cooperative Oncology Group.

\section{Patients and Methods}

Patient eligibility. The eligibility criteria included i) histopathologically proven colonic or rectal cancer, ii) clinical diagnosis of pulmonary metastasis from colorectal cancer, iii) no history of radiotherapy for the same lesion, iv) less than 80 years of age, v) Eastern Cooperative Oncology Group (ECOG) performance status of 2 or less, vi) no other active malignant tumor, and vii) absence of severe lung, heart or liver disorder.

Data for 104 lesions in 93 patients (60 males and 33 females) who received SBRT for pulmonary oligometastases from colorectal cancer between January 2004 and December 2013 at 10 institutions, which took part in the Japanese Radiation Oncology Study Group, were used for analysis in this retrospective study.

Toxicity. Toxicity was graded according to the Common Terminology Criteria for Adverse Events (CTCAE v4.0) (6).

Statistical analysis. Survival estimates were calculated using the Kaplan-Meier method from the first date of SBRT, and differences were evaluated by the log-rank test. Cox's hazard regression analysis using a backward stepwise selection model was used for multivariate analysis.

A $p$-value of less than 0.05 was considered significant. All analyses were performed using SPSS 22.0 (IBM Corp., Armonk, NY, USA).
Table II. Treatment characteristics.

\begin{tabular}{lc}
\hline Characteristic & N \\
\hline Prescribed dose & \\
$\geq 130$ Gy BED & 29 \\
$129-100$ Gy BED & 59 \\
$<100$ Gy BED & 16 \\
Prescribed method & \\
Isocenter & 77 \\
D95 & 24 \\
Unknown & 3 \\
Treatment period & \\
$<7$ Days & 80 \\
$\geq 7$ Days & 24 \\
Adjuvant chemotherapy & \\
Oral fluoropyrimidine & 22 \\
FOLFOX/FOLFIRI/XELOX & 9 \\
Other & 16 \\
None & 57 \\
\hline
\end{tabular}

BED: Biological effective dose.

Ethics. This retrospective study was approved by the Ethical Committees at all institutions.

\section{Results}

The median age of the patients was 69 years. The characteristics of the 93 patients with 104 lesions are shown in Table I. All patients completed SBRT. The median total irradiation dose was 50 Gy (range=40-65 Gy) per 3-15 fractions. The median calculated biological effective dose using the Linear-Quadratic (LQ) model with $\alpha / \beta=10$ Gy (BED10) was $105.6 \mathrm{~Gy}$ (range=72-134.4 Gy). The prescribed dose was delivered to the isocenter in 77 patients, covering $95 \%$ of the planning target volume (PTV) (D95) in 24 patients and by other methods in three patients. The median disease-free interval (DFI) between initial therapy for the primary site and SBRT was 18.2 months (range=0-112.5 months). Adjuvant chemotherapy after SBRT was performed in 47 patients. As adjuvant chemotherapy, oral fluoropyrimidines were used in 22 patients [uracil and tegafur (UFT) in eight patients, xeloda in two, S-1 in 10 patients and capecitabine in two], oxaliplatin, leucovorin and 5-fluorouracil (FOLFOX), irinotecan, leucovorin and 5-fluorouracil (FOLFIRI) or capecitabine and oxaliplatin (XELOX) was used in nine patients and other regimens or unknown regimens were used in the other patients. Treatment characteristics are shown in Table II.

During the observation period, 62 patients had recurrence or other metastases after SBRT and 48 patients died. The median observation period for 42 survivors was 28 months. The 3 - and 5-year overall survival rates were 55.9\% [95\% confidence interval $(\mathrm{CI})=43.7-68.1 \%]$ and $42.7 \%(95 \% \mathrm{CI}=28.8-56.7 \%)$, 


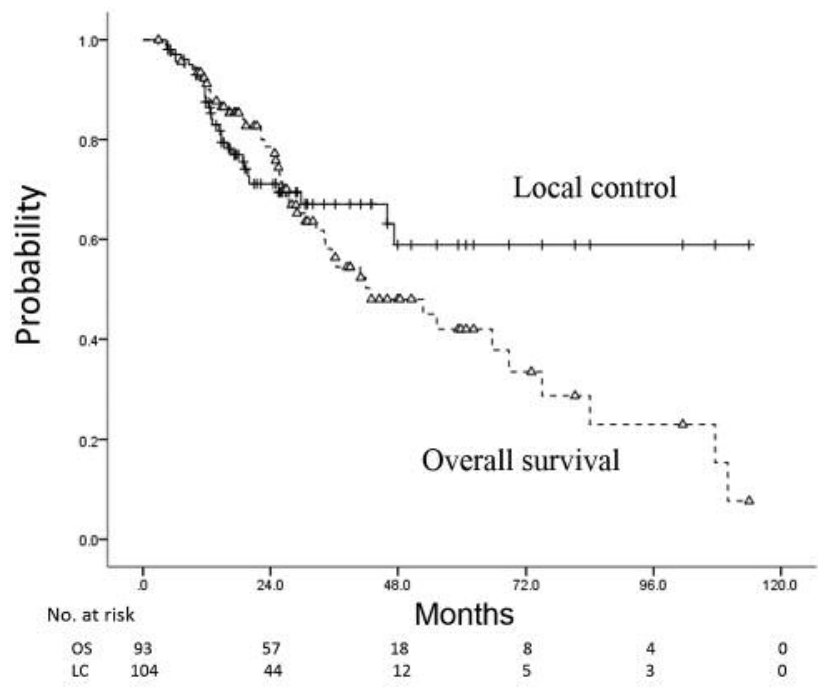

Figure 1. Overall survival rate and local control rate as determined by Kaplan-Meier methods.

respectively. The 3- and 5-year local control rates were $65.2 \%$ (95\% CI=53.8-76.6\%) and 56.2\% (95\% CI=41.1-71.3\%), respectively (Figure 1). The local control rate in patients who were treated with higher BED $(\geq 100$ Gy BED in patients prescribed with D95 or $\geq 130$ Gy BED10 in patients prescribed with an isocenter dose) was significantly better than that in patients treated with lower BED10 $(<100$ Gy BED in patients prescribed with D95 or $<130$ Gy BED in patients prescribed with an isocenter dose) (log-rank test, $p=0.011$ ) (Figure 2).

Only two patients had grade 3 radiation pneumonitis, including a patient with grade 5 . There was no other patient with grade 3 or higher toxicity.

In multivariate analysis for local control, primary site (rectal cancer being favorable) [hazard ratio $(\mathrm{HR})=0.375$, $95 \% \mathrm{CI}=0.159-0.884, p=0.025$ ], age (70 or more years being favorable) $(\mathrm{HR}=0.416,95 \% \mathrm{CI}=0.177-0.979, p=0.044)$, adjuvant chemotherapy after SBRT $(\mathrm{HR}=0.246,95 \%$ $\mathrm{CI}=0.097-0.625, p=0.003)$ and BED10 ( $\geq 100 \mathrm{~Gy}$ BED in patients prescribed with D95 or $\geq 130$ Gy BED in patients prescribed with an isocenter dose being favorable) $(\mathrm{HR}=0.100,95 \% \mathrm{CI}=0.013-0.768, p=0.027)$ were selected as prognostic factors. In multivariate analysis for overall survival, there was no significant prognostic factor.

\section{Discussion}

Local recurrence rates following resection of pulmonary oligometastases from colorectal cancer have been reported to be in the area of $20-30 \%$ (7-9). Retrospective analyses of radiofrequency ablation (RFA) for pulmonary metastases

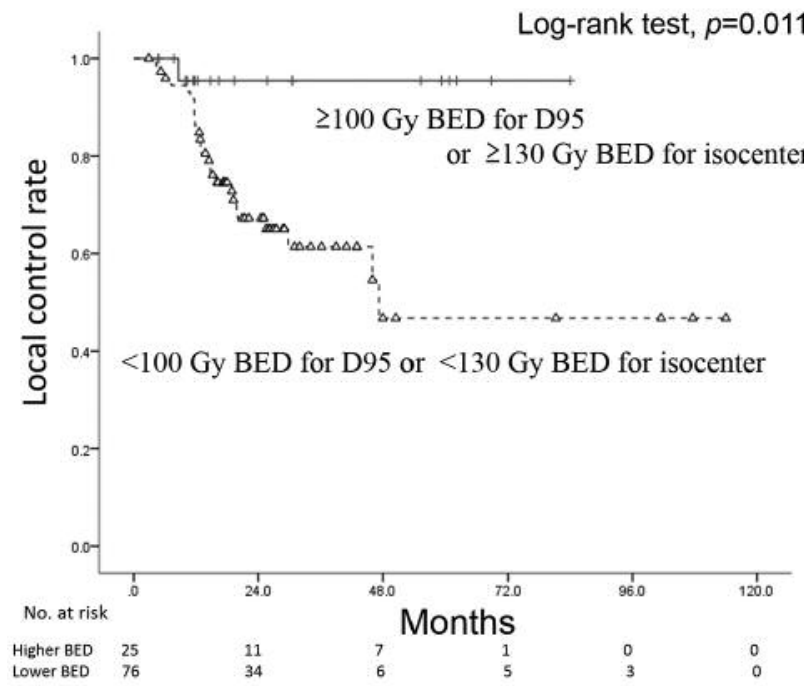

Figure 2. Local control rates achieved using higher and lower biological effective dose (BED) $(\geq 100$ Gy BED in patients prescribed with $D 95$ or $\geq 130$ Gy BED in patients prescribed with an isocenter dose being significantly favorable) as determined by Kaplan-Meier methods.

from colorectal cancer showed that the 2-year local control rate was $56-80 \%$ and the 2 -year overall survival rate was $34-$ $68 \%(10-12)$ (Table III).

To the best of our knowledge, this is the largest study of SBRT for pulmonary oligometastases from colorectal cancer at multiple Institutions. Despite the fact that almost of patients enrolled in this study were medically inoperable, overall survival and local control were comparable with those of past reports on resection or RFA. Especially in patients treated with higher irradiation dose, the local control rate was favorable. The results of a previous study showed that dose escalation might improve local control of liver metastases from colorectal cancer (13), and our results are consistent with this finding.

For $\geq 130$ Gy BED prescribed with the isocenter, standard prescribed doses for primary lung cancer in Japan (e.g. $45 \mathrm{~Gy} / 3,48 \mathrm{~Gy} / 4$ or $60 \mathrm{~Gy} / 8$ fractions) are insufficient (1416). It is necessary to add one or two fractions with the same prescribed dose to control pulmonary oligometastases. In the present study, there were few patients with severe toxicities caused by SBRT, and SBRT is considered to be an established treatment method for pulmonary tumors. However, dose escalation to improve local control may result in a higher incidence of severe toxicities, especially for metastases in the central lung zone, i.e. within a $2-\mathrm{cm}$ perimeter around the proximal bronchial tree. Therefore, the method with the prescribed dose being $60 \%$ of the maximal dose in PTV reported by Takeda et al. might be better than that at D95 (17). 
Table III. Literature review of treatment results for lung metastases from colorectal cancer.

\begin{tabular}{|c|c|c|c|c|c|}
\hline Authors & Year & Treatment method & $\mathrm{N}$ & Local recurrence & Overall survival \\
\hline Welter et al. (7) & 2007 & Surgery & 169 & n.a. & 5-Year: $39.1 \%$ \\
\hline De Giacomo et al. (8) & 1999 & Surgery & 24 & $21.7 \%$ & 5-Year: $49.5 \%$ \\
\hline Landreneau et al. (9) & 2000 & Surgery & 80 & $8 \%$ & 5-Year: $30.8 \%$ \\
\hline Yan et al. (10) & 2007 & RFA & 55 & $38 \%$ & 3-Year: $46 \%$ \\
\hline Yamakado et al. (11) & 2009 & RFA & 140 & 5-Year: $20.6 \%$ & 5-Year: $57.0 \%$ \\
\hline Chua et al. (12) & 2010 & RFA & 100 & n.a. & 3-Year: $50 \%$ \\
\hline Current study & 2016 & SBRT & 103 & 3-Year: $34.8 \%$ & 3-Year: $55.9 \%$ \\
\hline
\end{tabular}

RFA: Radiofrequency ablation.

The fact that adjuvant chemotherapy improved local control is reasonable. Adjuvant or neoadjuvant chemotherapy with surgery for pulmonary metastases from colorectal cancer has already been shown to have a good effect (18); however, to our knowledge, this is the first report to show the effect of adjuvant chemotherapy after SBRT. Unfortunately, a beneficial effect on survival was not shown. This might be due to the inclusion of patients who had resistance to chemotherapy or due to the inclusion of various chemotherapy regimens. A prospective study is needed to determine the appropriate regimen after SBRT.

Our results suggest that local control of oligometastases from rectal cancer was significantly better than that of oligometastases from colonic cancer. It is known that outcome in patients with metastases from right-sided colonic cancer is worse than that from left-sided colonic and rectal cancer (19); this may be due to molecular differences (e.g. KRAS and BRAF status, and microsatellite instability). However, to the best of our knowledge, this is the first report to show that the local control rate with SBRT for lung metastases from rectal cancer is better than that from colonic cancer. Although we do not know why there was no significant difference in the overall survival rate, our findings suggest that metastases from colonic cancer may have greater resistance to radiation compared with metastases from rectal cancer.

The concept of oligometastases was first proposed by Hellman et al. in 1995 (20). Niibe et al. broke-down the concept of oligometastases into oligo-recurrence and syncoligometastases (21). The major difference between oligorecurrence and sync-oligometastases is the status of the primary lesion, which is the most important prognostic factor of oligometastases, and oligo-recurrence conferrered better prognosis than sync-oligometastases for certain kinds of malignant tumors. The status of patients with more than 6 months of DFI in the present study was oligo-recurrence; however, there was no significant difference in the local control rate or overall survival rate even by log-rank test. In metastasectomy of colorectal cancer, DFI was reported as a prognostic factor (22). This difference might be due to chemotherapy before SBRT.

Unfortunately there exist some limitations to this study. It was a retrospective study, with various treatment methods. In many patients with colonic cancer, primary site details (e.g. sigmoid, ascending descending and transverse) were unknown.

\section{Conclusion}

The efficacy of SBRT for pulmonary metastasis from colorectal cancer is not sufficient; however, dose escalation and adjuvant chemotherapy might improve local control.

\section{Conflicts of Interest}

None declared.

\section{References}

1 Hirosawa T, Itabashi M, Ohnuki T, Yamaguchi N, Sugihara K, Kameoka $\mathrm{S}$ and Japanese Society for Cancer of the Colon and Rectum Study Group for Pulmonary Metastases from Colorectal Cancer: Proposal of a new prognostic staging system for pulmonary metastases from colorectal cancer. Surg Today 45(5): 576-584, 2015.

2 Pfannschmidt J, Dienemann $\mathrm{H}$ and Hoffmann H: Surgical resection of pulmonary metastases from colorectal cancer: a systematic review of published series. Ann Thorac Surg 84: 324338, 2007.

3 National Comprehensive Cancer Network: Practice Guidelines in Oncology for Colon Cancer. v.2. 2017. https://www.ncen.org/ professionals/physician_gls/pdf/colon.pdf

4 Yamamoto T, Jingu K, Shirata Y, Koto M, Matsushita H, Sugawara T, Kubozono M, Umezawa R, Abe K, Ishikawa Y, Kozumi M, Takahashi N, Takeda K and Takai Y: Outcomes after stereotactic body radiotherapy for lung tumors, with emphasis on comparison of primary lung cancer and metastatic lung tumors. BMC Cancer 14: 464, 2014.

5 Aoki M, Hatayama Y, Kawaguchi H, Hirose K, Akimoto H, Miura H, Ono S and Takai Y: Stereotactic body radiotherapy for lung metastases as oligo-recurrence: a single institutional study. J Radiat Res 57(1): 55-61, 2016. 
6 http://ctep.cancer.gov/protocolDevelopment/electronic_appli cations/ctc.htm\#ctc_40

7 Welter S, Jacobs J, Krbek T, Poettgen C and Stamatis G: Prognostic impact of lymph node involvement in pulmonary metastases from colorectal cancer. Eur J Cardiothorac Surg 31(2): 167-172, 2007.

8 De Giacomo T, Rendina EA, Venuta F, Ciccone AM and Coloni GF: Thoracoscopic resection of solitary lung metastases from colorectal cancer is a viable therapeutic option. Chest 115: 14411443, 1999.

9 Landreneau RJ, De Giacomo T, Mack MJ, Hazelrigg SR, Ferson PF, Keenan RJ, Luketich JD, Yim AP and Coloni GF: Therapeutic video-assisted thoracoscopic surgical resection of colorectal pulmonary metastases. Eur J Cardiothorac Surg 18(6): 671-676, 2000.

10 Yan TD, King J, Sjarif A, Hazelrigg SR, Ferson PF, Keenan RJ, Luketich JD, Yim AP and Coloni GF: Treatment failure after percutaneous radiofrequency ablation for nonsurgical candidates with pulmonary metastases from colorectal carcinoma. Ann Surg Oncol 14: 1718-1726, 2007.

11 Yamakado K, Inoue Y, Takao M, Takaki H, Nakatsuka A, Uraki J, Kashima M, Kusunoki M, Shimpo H and Takeda K: Longterm results of radiofrequency ablation in colorectal lung metastases: single center experience. Oncol Rep 22: 885-891, 2009.

12 Chua TC, Thornbury K, Saxena A, Liauw W, Glenn D, Zhao J and Morris DL: Radiofrequency ablation as an adjunct to systemic chemotherapy for colorectal pulmonary metastases. Cancer 116: 2106-2114, 2010.

13 Lausch A, Sinclair K, Lock M, Fisher B, Jensen N, Gaede S, Chen $\mathrm{J}$ and Wong E: Determination and comparison of radiotherapy dose responses for hepatocellular carcinoma and metastatic colorectal liver tumours. Br J Radiol 86: 20130147, 2013.

14 Nagata Y, Hiraoka M, Shibata T, Onishi H, Kokubo M, Karasawa K, Shioyama Y, Onimaru R, Kozuka T, Kunieda E, Saito T, Nakagawa K, Hareyama M, Takai Y, Hayakawa K, Mitsuhashi N and Ishikura S: Prospective trial of stereotactic body radiation therapy for both operable and inoperable T1N0M0 non-small cell lung cancer: Japan Clinical Oncology Group Study JCOG0403. Int J Radiat Oncol Biol Phys 93(5): 989-996, 2015.
15 Onimaru R, Shirato H, Shimizu S, Kitamura K, Xu B, Fukumoto S, Chang TC, Fujita K, Oita M, Miyasaka K, Nishimura M and Dosaka-Akita $\mathrm{H}$ : Tolerance of organs at risk in small-volume, hypofractionated, image-guided radiotherapy for primary and metastatic lung cancers. Int J Radiat Oncol Biol Phys 56(1): 126-135, 2003

16 Koto M, Takai Y, Ogawa Y, Matsushita H, Takeda K, Takahashi C, Britton KR, Jingu K, Takai K, Mitsuya M, Nemoto K and Yamada S: A phase II study on stereotactic body radiotherapy for stage I non-small cell lung cancer. Radiother Oncol 85(3): 429-434, 2007.

17 Oku Y, Takeda A, Kunieda E, Sudo Y, Oooka Y, Aoki Y, Shimouchi Y, Nishina R, Nomura K, Sugiura M and Ohashi T: Analysis of suitable prescribed isodose line fitting to planning target volume in stereotacticbody radiotherapy using dynamic conformal multiple arc therapy. Pract Radiat Oncol 2(1): 46-53, 2012.

18 Brandi G, Derenzini E, Falcone A, Masi G, Loupakis F, Pietrabissa A, Pinna AD, Ercolani G, Pantaleo MA, Di Girolamo S, Grazi GL, de Rosa F and Biasco G: Adjuvant systemic chemotherapy after putative curative resection of colorectal liver and lung metastases. Clin Colorectal Cancer 12(3): 188-194, 2013.

19 Price TJ, Beeke C, Ullah S, Padbury R, Maddern G, Roder D, Townsend AR, Moore J, Roy A, Tomita Y and Karapetis C: Does the primary site of colorectal cancer impact outcomes for patients with metastatic disease? Cancer 121(6): 830-835, 2015.

20 Hellman S and Weichselbaum RR: Oligometastases. J Clin Oncol 13: 8-10, 1995.

21 Niibe Y and Chang JY: Novel insights of oligometastases and oligo-recurrence and review of the literature. Pulm Med 2012: 261096, 2012.

22 Kim S, Kim HK, Cho JH, Choi YS, Kim K, Kim J, Zo JI, Shim YM, Heo JS, Lee WY and Kim HC: Prognostic factors after pulmonary metastasectomy of colorectal cancers: influence of liver metastasis. World J Surg Oncol 14(1): 201, 2016.

Received March 7, 2017

Revised March 24, 2017

Accepted March 31, 2017 